Supporting Information for

\title{
Synthesis of photoreactive poly(ethylene oxide)s for
}

\section{surface modification}

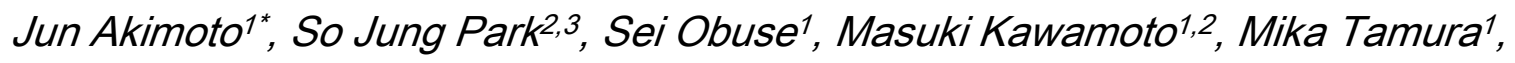

Avanashiappan Nandakumar', Eiry Kobatake ${ }^{3}$, and Yoshihiro Ito ${ }^{1,2^{*}}$

${ }^{1}$ Emergent Bioengineering Materials Research Team, RIKEN Center for Emergent

Matter Science, 2-1 Hirosawa, Wako, Saitama 351-0198, Japan

${ }^{2}$ Nano Medical Engineering Laboratory, RIKEN Center for Pioneering Research, 2-1

Hirosawa, Wako, Saitama 351-0198, Japan

${ }^{3}$ Department of Life Science and Technology, School of Life Science and Technology,

Tokyo Institute of Technology, 4259 Nagatsuda-cho, Midori-ku 226-8502, Yokohama, Japan 


\section{Corresponding Authors}

*E-mail: jun.akimoto@riken.jp (J.A.)

*E-mail: y-ito@riken.jp (Y.I.)

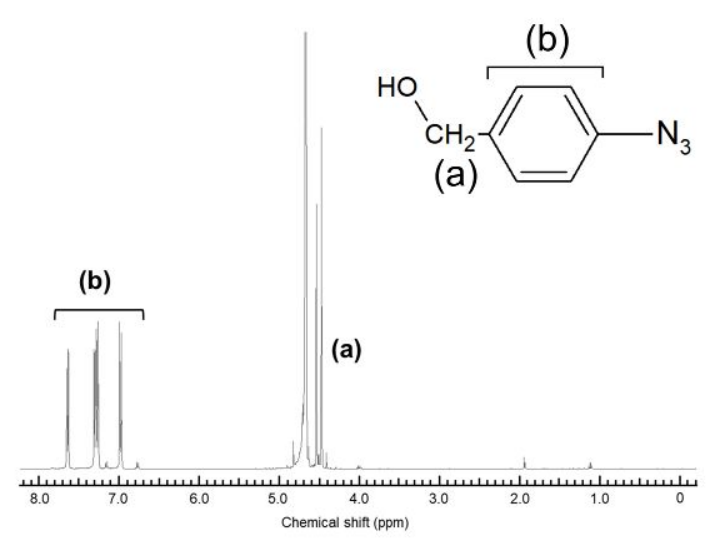

Figure $\mathrm{S} 1 .{ }^{1} \mathrm{H}$ NMR spectrum of 4-(hydroxymethyl)azidobenzene (Solvent: $\mathrm{D}_{2} \mathrm{O}$ ).

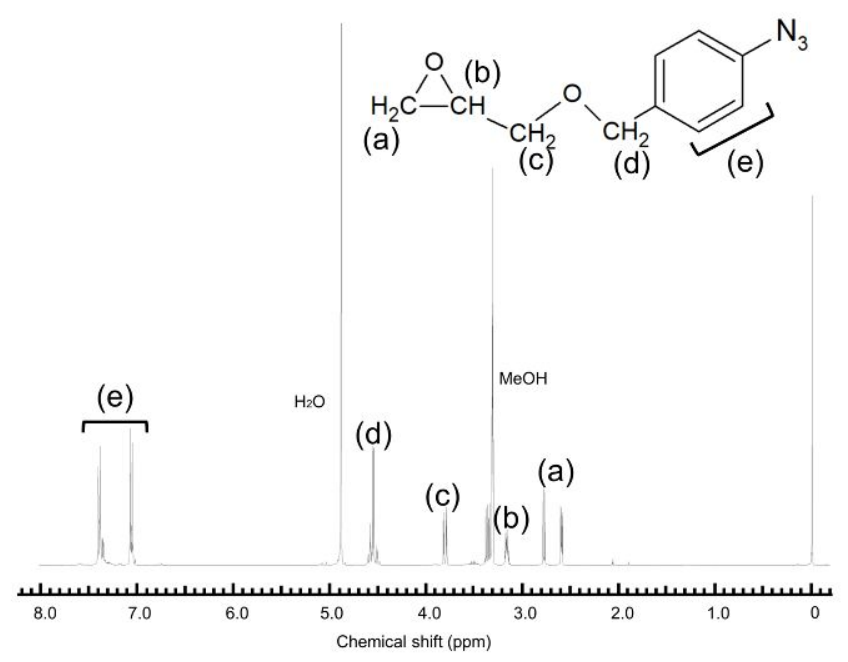


Figure S2. ${ }^{1} \mathrm{H}$ NMR spectrum of 4-(glycidyloxymethyl)azidobenzene (Solvent: methanol$\left.\mathrm{d}_{4}\right)$.

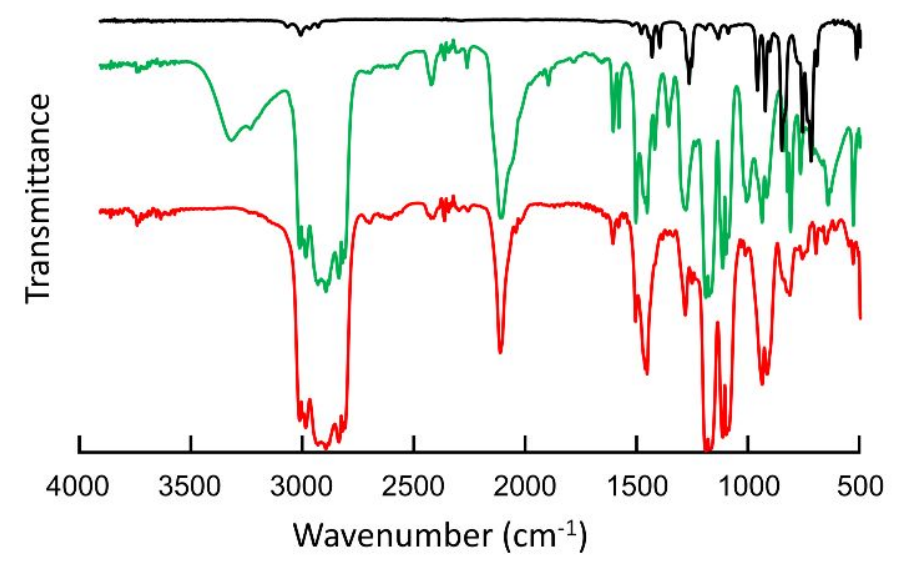

Figure S3. FT-IR spectra of epichlorohydrin (black), 4-(hydroxymethyl)azidobenzene (green), and 4-(glycidyloxymethyl)azidobenzene (red).

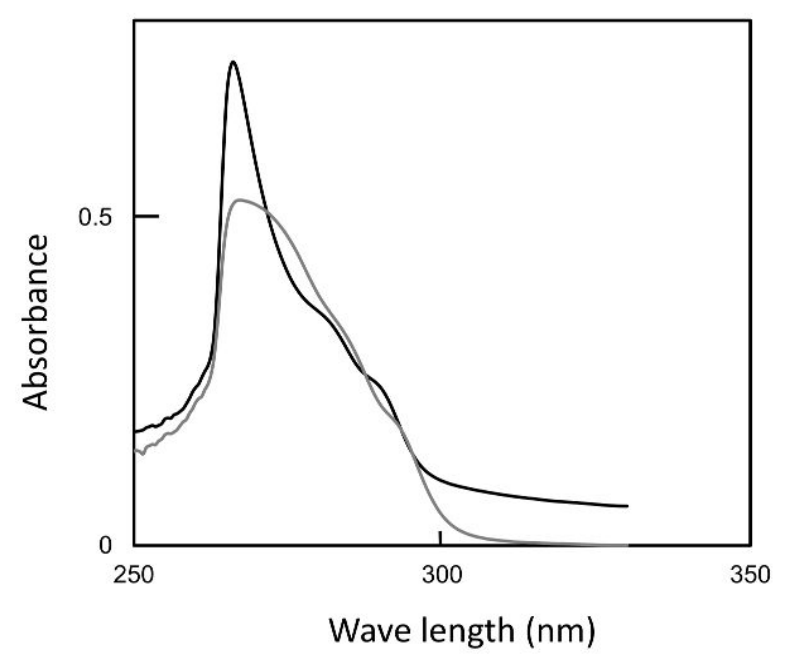

Figure S4. UV absorbance spectra of AzPEG(9) (black) and azidobenzoic acid (gray) in DMF. 


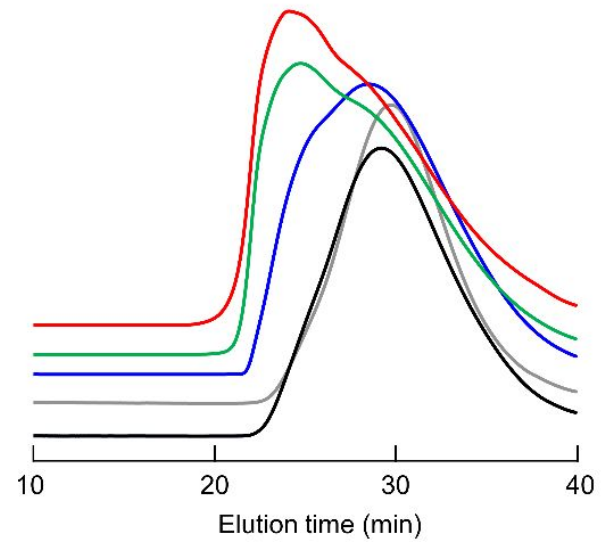

Figure S5. GPC traces of AzPEG(1.2) (black), AzPEG(3) (gray), AzPEG(9) (blue), AzPEG(14) (green), and AzPEG(30) (red). Eluent: DMF containing $10 \mathrm{mmol} / \mathrm{L} \mathrm{LiBr}$.

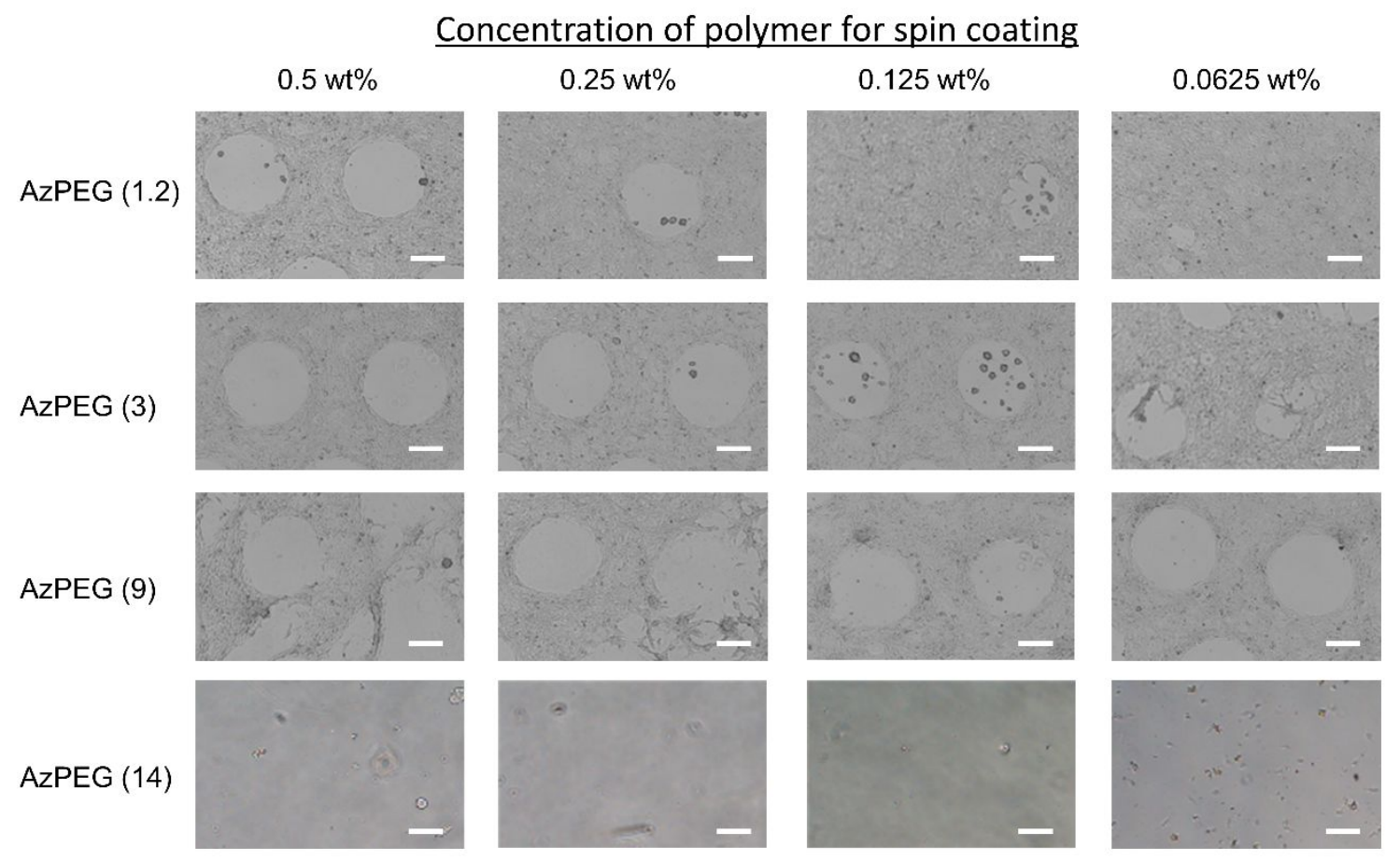

Figure S6. Phase contrast images of 3T3 cultured on AzPEG-coated micro-patterned glass. Effect of composition: the surfaces were rinsed with water after photoimmobilization. Scale bar: $200 \mu \mathrm{m}$. 


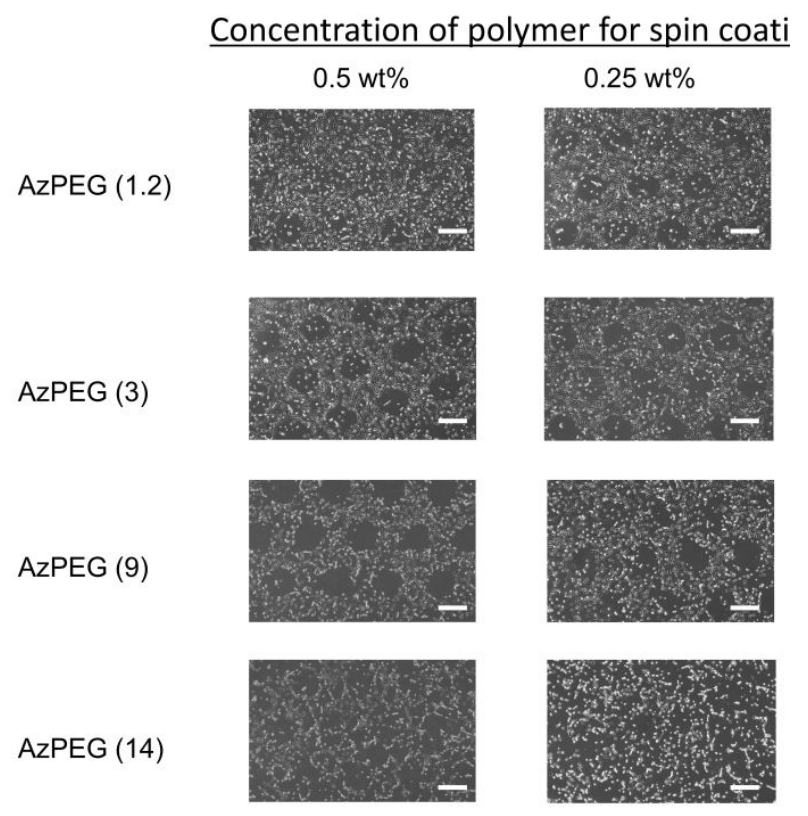

Figure S7. Phase contrast images of 3 T3 cultured on AzPEG-coated micro-patterned polystyrene dish. Seeding density $=5.0 \times 10^{3}$ cells $/ \mathrm{cm}^{2}$. Incubation time $=24 \mathrm{~h}$. Scale bars: $500 \mu \mathrm{m}$.

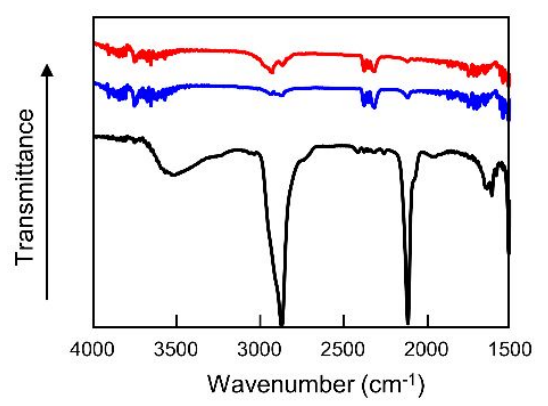

Figure S8. FTIR spectra of AzPEG(9)-coated glass surfaces. Traces are before (black) and after rinsing with water (blue) and methanol (red). 


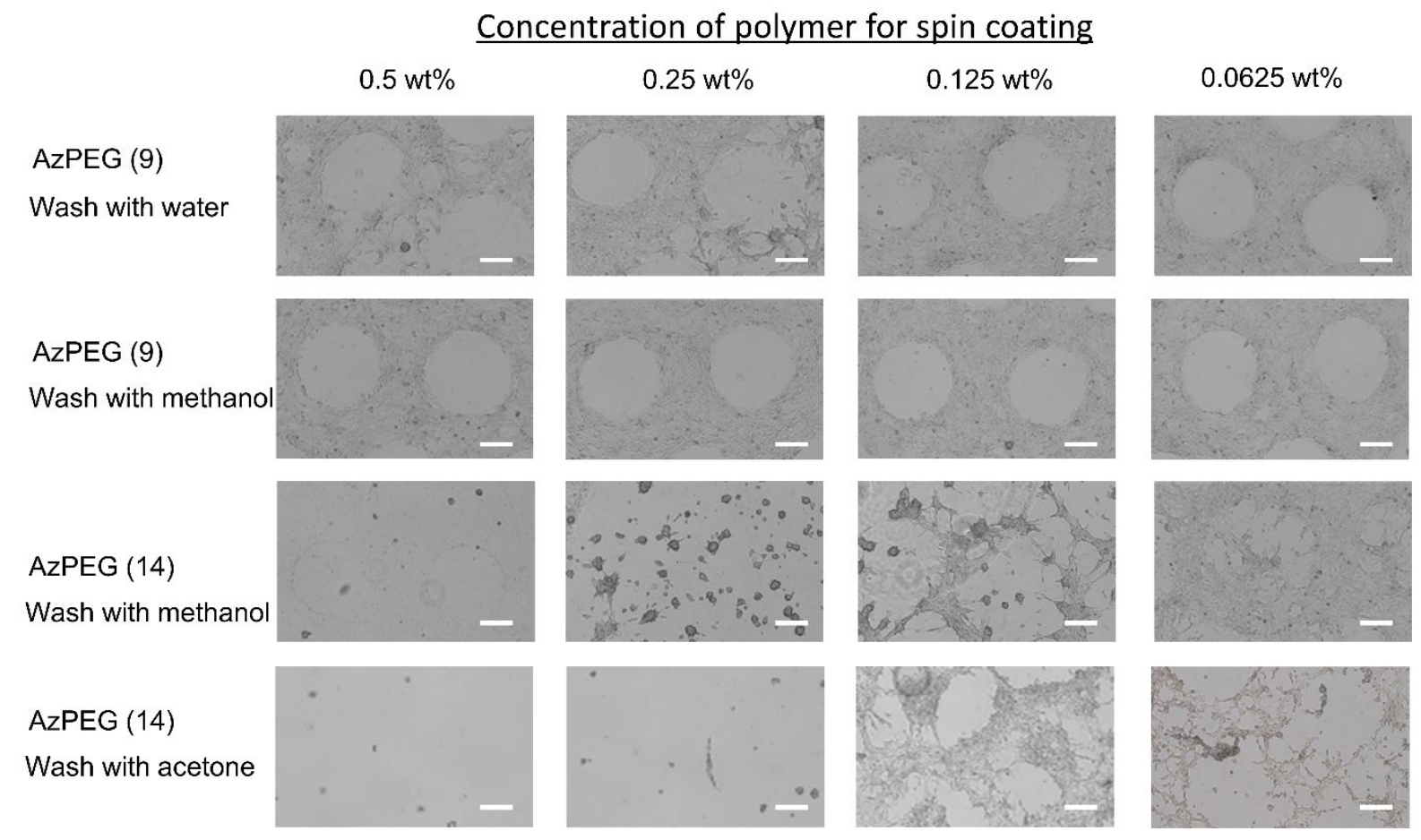

Figure S9. Phase contrast images of 3 T3 cultured on AzPEG-coated micro-patterned glass. The effect of washing solution: the surfaces were rinsed with water, methanol, or acetone after photo-immobilization. Scale bar: $200 \mu \mathrm{m}$.

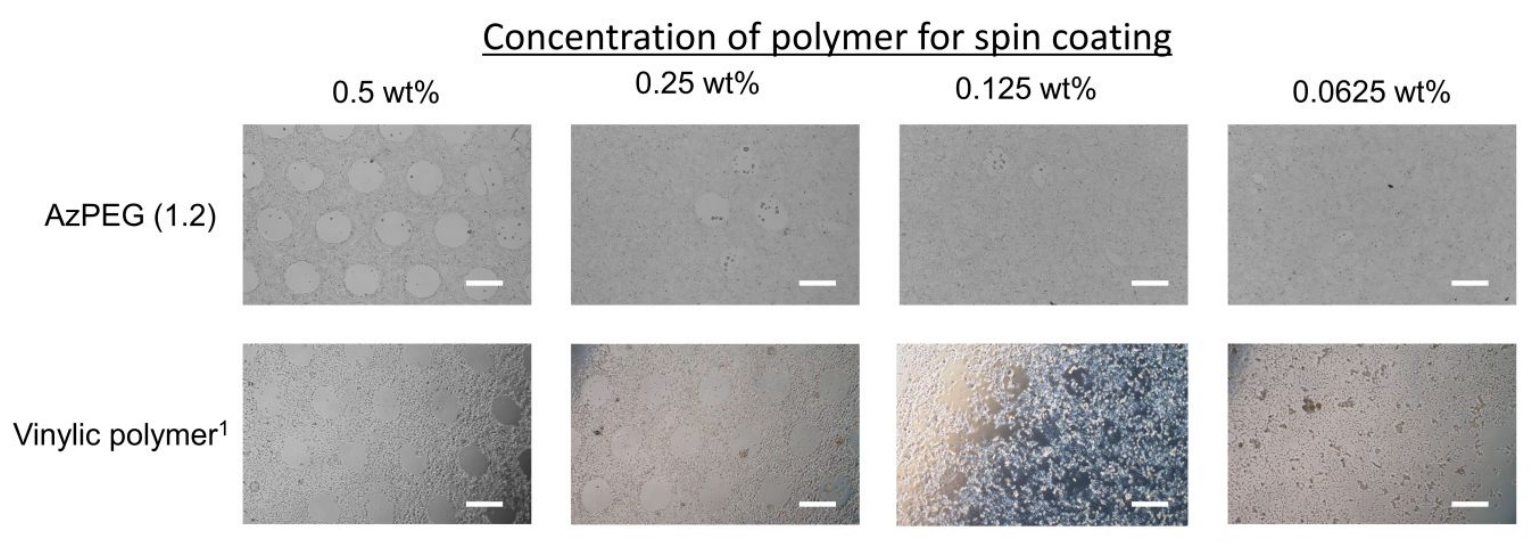

Figure S10. Phase contrast images of 3T3 cultured on AzPEG(1.2)- and vinylic-polymercoated micro-patterned glass. The surfaces were rinsed with water. The vinylic polymer is poly(pentaethylene glycol methacrylate) ${ }_{0.9}$-co-poly(azidobenzyl methacrylate $)_{0.1}$. Scale bar: $500 \mu \mathrm{m}$. 
